\title{
Interplay of network dynamics and heterogeneity of ties on spreading dynamics
}

\author{
Luca Ferreri, ${ }^{1,2, *}$ Paolo Bajardi, ${ }^{1,2}$ Mario Giacobini,${ }^{1,2,3}$ Silvia Perazzo, ${ }^{4}$ and Ezio Venturino ${ }^{4}$ \\ ${ }^{1}$ GECO-Computational Epidemiology Group, Department of Veterinary Sciences, University of Torino, largo Braccini 2 , \\ IT-10095 Grugliasco (TO) \\ ${ }^{2}$ ARCS - Applied Research on Computational Complex Systems Group, Department of Computer Science, \\ University of Torino, corso Svizzera 185, IT-10149 Torino \\ ${ }^{3}$ CSU-Complex Systems Unit, Molecular Biotechnology Centre, University of Torino, via Nizza 52, IT-10126 Torino \\ ${ }^{4}$ Department of Mathematics "Giuseppe Peano", University of Torino, via Carlo Alberto 10, IT-10123 Torino
}

(Received 10 September 2013; revised manuscript received 22 May 2014; published 28 July 2014)

\begin{abstract}
The structure of a network dramatically affects the spreading phenomena unfolding upon it. The contact distribution of the nodes has long been recognized as the key ingredient in influencing the outbreak events. However, limited knowledge is currently available on the role of the weight of the edges on the persistence of a pathogen. At the same time, recent works showed a strong influence of temporal network dynamics on disease spreading. In this work we provide an analytical understanding, corroborated by numerical simulations, about the conditions for infected stable state in weighted networks. In particular, we reveal the role of heterogeneity of edge weights and of the dynamic assignment of weights on the ties in the network in driving the spread of the epidemic. In this context we show that when weights are dynamically assigned to ties in the network, a heterogeneous distribution is able to hamper the diffusion of the disease, contrary to what happens when weights are fixed in time.
\end{abstract}

DOI: 10.1103/PhysRevE.90.012812

PACS number(s): 89.75.Hc, 05.45.Xt, 87.23.Ge

\section{INTRODUCTION}

In the last decade, an increasing scientific effort has been devoted to the understanding and characterization of spreading phenomena in complex settings, ranging from computer viruses to rumors, human diseases, and animals pathogens. To this aim, the study of diffusion processes on complex networks represents a major contribution to move beyond the homogeneous mixing approximation and to investigate the effect of the interaction of hosts in shaping the epidemic spreading. Heterogeneous networks are usually described as annealed or quenched. While an annealed network is defined by an adjacency matrix fixed only on average [1], the latter is defined by a fixed adjacency matrix. In this context, until today, there have been two main analytical approaches that could be exploited to describe dynamical process occurring on heterogeneous networks: the heterogeneous mean-field (HMF) theory and the spectral approach [2,3]. More specifically, the HMF approach is suitable for annealed networks [1] and predicts an epidemic threshold inversely proportional to the second moment of the network's degree distribution. In addition, the HMF approach is also able to capture the epidemic threshold for some classes of quenched networks (QN), for instance when the degree is power-law distributed with an exponent between 2 and 2.5 [3]. However, it has been demonstrated that the phase transition of QN is properly described by the spectral radius of the adjacency matrix [4-7]. Both approaches pointed out the dramatic effects of contact heterogeneities in lowering the epidemic threshold [3,8-11]. Beyond the degree centrality, other measures have been recognized to be important indicators of the role of a node in the diffusion process, such as the betweenness centrality $[12,13]$, closeness

\footnotetext{
*luca.ferreri@unito.it
}

[14,15], eigenvector and PageRank [16,17], and $k$ shell [18-20].

At the same time, great interest has arisen in the last years in understanding and exploring evolving graphs [21-25] and their interplay with dynamical process occurring on them. Schwarzkopf and colleagues [26], for instance, introduced and analyzed a model for epidemic spreading on a rewiring network, highlighting that the rewiring process tends to suppress the infection. Similarly, Volz and his collaborators [27-29] worked on the impact of the contact duration on the pathogen's diffusion. Meanwhile, other models have been proposed describing the coevolution of the graph and the spreading process [30-35], showing the nonlinear interplay of the two dynamics. More recently, Perra and collaborators [36], introduced an activity driven model in which the instantaneous interaction of agents is defined by the activity potential and provided an analytical description of the epidemic threshold in such context. In particular, via an HMF approximation, they showed that the stable infected state depends on the first and second moments of the nodes' activity distribution probability.

While many researches have been conducted on unweighted graphs up today, a limited knowledge [37-46] is currently available on the effect of the weight of the links on pathogen spreading over an evolving graph. This is a relevant issue for exploring real-world scenarios, where dynamical contacts between hosts are observed [47-50] and a weighted representation is often required [51-55]. In this paper we are interested in understanding how the combination of heterogeneity of edge weights and the dynamic assignment of weights on network's ties could affect the epidemic spreading. From an epidemiological point of view, we can interpret weights on ties as an indicator of the intensity or duration of the interactions between two connected nodes representing the hosts of the pathogen. There exist several examples in realworld settings where a weighted representation of contacts between network nodes is needed [56]: from the early work on 
sociograms [57], where weights describe the number of real contacts among two people, to email networks, where weights represent the number of exchanged emails, to cattle trade movements, where weights take into account the number of animals moved between livestock premises [53]. Although the intuition and common knowledge suggest that heavier weights should indicate greater ease of transmission, a comprehensive understanding of the system dynamics is still lacking. To bridge the gap between intuition and formal investigation, and to extend our findings to dynamical networks, we define a theoretical and manageable framework to perform both analytical calculations and computational experiments.

\section{GRAPHS WITH STABLE WEIGHTS PATTERN}

\section{A. Graphs generation}

As a first step in analyzing the role of edge weights in an epidemic spreading, we need a series of networks with some desired properties: an assigned degree distribution, the same number of edges, an assigned weight heterogeneity, and the total amount of weights. To this end we work on graphs of size $N=10^{4}$. We initially create a sequence of stubs by pooling from a probability distribution $p(k) \sim k^{-2.25}$ (we choose as exponent of the power law 2.25, a good compromise between induced variability and a sufficiently large average degree $\langle k\rangle \simeq 5$ ), and then we tune the degree heterogeneity of the produced network by randomly reshuffling a fraction $\gamma_{k} \in[0,1]$ of the stubs' origins. We would like to underline that when $\gamma_{k}=1$ all the stubs' origins are reshuffled, resulting in a random assignment of contacts, while for $\gamma_{k}=0$ the network exhibits the maximum degree of heterogeneity as caused by exponent 2.25. Since the reshuffling is performed on the open stubs and not on the links, the reshuffling process destroys the original degree sequence of the nodes, thus defining the new network topology.

We further remind that a vanishing epidemic threshold is observed when the degree is power-law distributed with an exponent between 2 and 3. Therefore we considered an exponent of 2.25 as our most heterogeneous case and, by increasing the proportion of rewired links, we systematically explored increasingly homogeneous systems. At the end of this procedure, we assigned a discrete weight to every stub by generating it from the probability distribution $q(w) \sim w^{-2.25}$. Again, we choose the exponent 2.25 because of its interplay between strong heterogeneities and not too small average edge weight $(\langle w\rangle \simeq 2$.66). In order to tune the heterogeneity of the weight distribution, similarly to the rewiring procedure described before, we randomly selected a fraction $\gamma_{w}$ of the allocated weights and randomly reassigned them so that the new $w_{i_{\bullet}}^{\prime}=w_{i_{\bullet}}-1$ and $w_{k_{\bullet}}^{\prime}=w_{k_{\bullet}}+1, w_{i \bullet}$ being the weight of a stub departing from node $i$. We further impose that stubs of weight 1 could not be reallocated in order to avoid stubs with zero weights and to keep the degree of the nodes constant. When examining a routing system or an infrastructural network with packets or hosts moving between nodes, reallocating a fraction of the total weight across the network allows us to tune the heterogeneity of the weight distribution while keeping the total amount of traffic and average weights constant. Then, using the configuration model

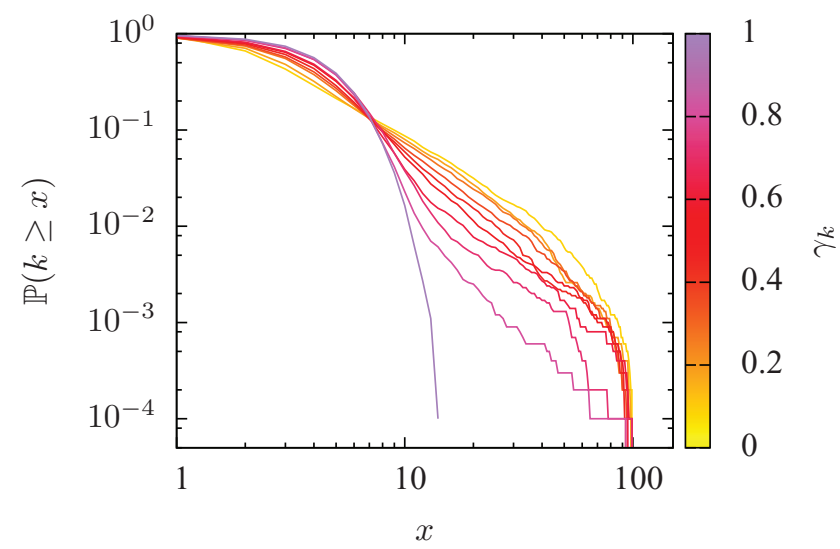

FIG. 1. (Color online) Degree distribution of networks varying $\gamma_{k}$ and maintaining $\gamma_{w}=0 . \gamma_{k}$ ranges from 0 , yellow (upper) curve, to 1 , violet (lower) curve.

[58], adjusted to generate uncorrelated networks [59], we close the stubs by further imposing that a stub of weight $w$ is tied with a stub of the same weight (i.e., $w_{i}$ being a stub departing from $i$ and having weight $w$, it can be connected with any stub $w_{j \bullet}$ where $j \neq i$ ). With the described algorithm, we are able to generate networks with the same number of edges and total traffic and the desired degree and weight heterogeneities. We refer to Figs. 1-3 for some graph features obtained with various $\gamma_{k}$ and $\gamma_{w}$. More in detail, we highlight that for $\gamma_{k}=0$ we let $k$ range between 2 and $\sqrt{N}[58,59]$, while for $\gamma_{w}=0$ we let $w$ range between 1 and $N$.

We further remark that in the following we are going to consider two classes of graph: (i) annealed, if the graph is defined only by the degree and weight sequences (i.e., the stubs are closed at each considered iterations); (ii) quenched, if the graph is the result of the stubs closure (i.e., the weighted adjacency matrix is fixed in time).

\section{B. Disease spreading}

In the following section we focus on the susceptibleinfectious-susceptible (SIS) compartmental model [60], where

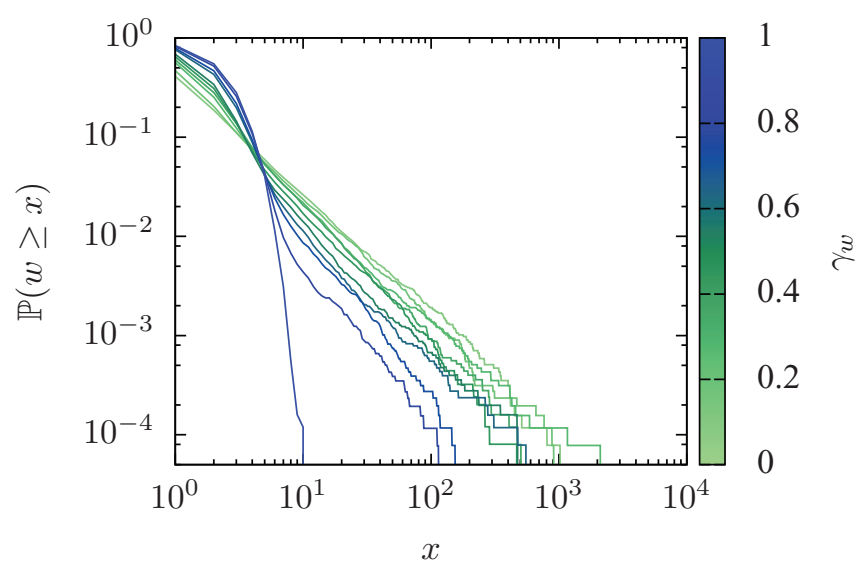

FIG. 2. (Color online) Weight distribution of networks varying $\gamma_{w}$ and maintaining $\gamma_{k}=0 . \gamma_{w}$ ranges from 0 , light-green (upper) curve, to 1 , blue (lower) curve. 


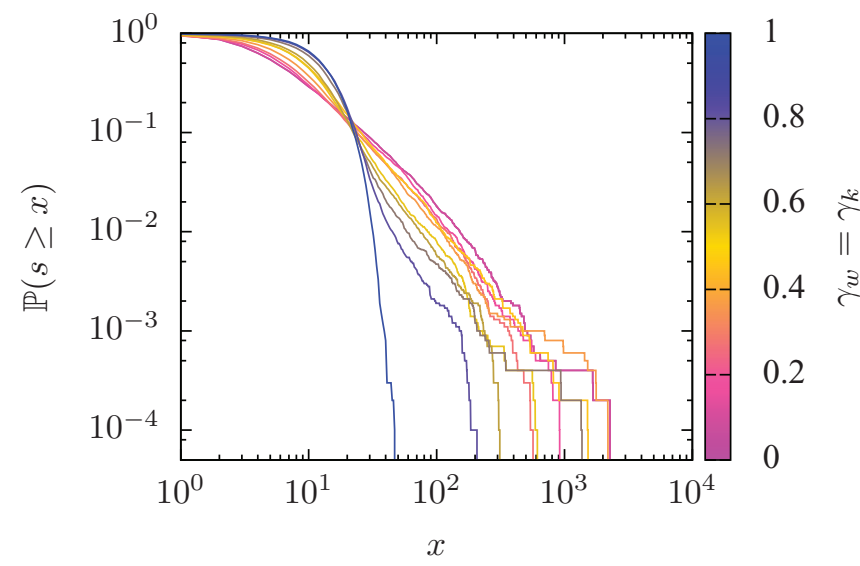

FIG. 3. (Color online) Strength distribution of networks varying $\gamma_{w}=\gamma_{k} \cdot \gamma_{w}=\gamma_{k}$ ranges from 0 , magenta (upper) curve, to 1 , blue (lower) curve.

nodes are divided in two classes according to their health status. A susceptible node is infected by an infective neighbor with probability $\beta$ and an infective node recovers from the infection with probability $\mu$, thus becoming susceptible again.

\section{Analytical description}

In order to achieve a qualitative understanding on the critical behavior of the spreading phenomena on weighted networks, we use an HMF approach [11] that can deal with weighted transmission routes. The HMF approach is known to be exact for weighted annealed networks [39] while for quenched networks a spectral approach should be preferred. Using a strength block approximation (i.e., assuming that all nodes with the same strength are statistically and epidemiologically equivalent), we can describe the variation of the fraction of infectious nodes of strength $s$ as

$$
\frac{d i_{s}}{d t}=-\mu i_{s}+\left(1-i_{s}\right)\left[1-(1-\beta)^{s \Theta_{s}}\right],
$$

where $\Theta_{s}$ is the probability that an edge originating from an infectious node is connected to a susceptible $s$ node. Furthermore, assuming no strength correlation among connected nodes we have that

$$
\Theta_{s}=\frac{1}{\langle s\rangle} \sum_{s^{\prime}} s^{\prime} P\left(s^{\prime}\right) i_{s^{\prime}}=\Theta,
$$

where $P\left(s^{\prime}\right)$ is the probability for a node to have strength $s^{\prime}$. Imposing the stability condition $\frac{d i_{s}}{d t}=0$, after some algebraic manipulations, we find

$$
\Theta=G(\Theta)=\frac{1}{\langle s\rangle} \sum_{s} s P(s) \frac{\left[1-(1-\beta)^{s \Theta}\right]}{\mu+\left[1-(1-\beta)^{s \Theta}\right]},
$$

which, at least to our knowledge, cannot be analytically solved. However, in the considered domain, we have that $G(\Theta) \leqslant$ $\Theta$ and $G(0)=0$. Moreover, $G$ is an increasing and concave function. Thus, if $G^{\prime}(0)>1$, there exists $\hat{\Theta} \in(0,1]$ such as $G(\hat{\Theta})=\hat{\Theta}$. In conclusion, a nonzero solution exists if

$$
-\frac{\ln (1-\beta)}{\mu}>\frac{\langle s\rangle}{\left\langle s^{2}\right\rangle} \text {. }
$$

Now, we want to understand how the strength changes in function of the weight and degree distribution, respectively $q(w)$ and $p(k)$. To this end, the probability generating function (pgf), [61], of the degree is

$$
A(x)=\sum_{k} x^{k} p(k),
$$

while $B(x)=\sum_{w} x^{w} q(w)$ is the pgf of the weight probability distribution. Consider now a node connected with $k$ other nodes. We define $S_{k}=w_{1}+\cdots+w_{k}$ as the random variables of the strength of a node of degree $k$. The pgf of such variables is

$$
\begin{aligned}
C(x, k) & =\sum_{z} x^{z} P\left(S_{k}=z\right) \\
& =\sum_{z} x^{z} P\left(w_{1}+\cdots+w_{k}=z\right) \\
& =\left(\sum_{h} x^{h} q(h)\right)^{k}=(B(x))^{k} .
\end{aligned}
$$

Now, if the degree of nodes is also random then the pgf of the strength of a node is

$$
\begin{aligned}
D(x) & =\sum_{s} x^{s} P(S=s)=\sum_{s} x^{s} \sum_{h} P\left(S_{h}=s\right) p(h) \\
& =\sum_{h} p(h) \sum_{s} x^{s} P\left(S_{h}=s\right)=\sum_{h} p(h) C(x, h) \\
& =\sum_{h} p(h)(B(x))^{h}=A(B(x)) .
\end{aligned}
$$

From a pgf, $G(x)$, the $n$th moment of the distribution can be calculated as follows: $\left\langle k^{n}\right\rangle=\left[\left(x \frac{d}{d x}\right)^{n} G(x)\right]_{x=1}$. Hence,

$$
\langle s\rangle=A^{\prime}(B(x=1)) B^{\prime}(x=1)=\langle k\rangle\langle w\rangle,
$$

and

$$
\begin{aligned}
\left\langle s^{2}\right\rangle & =A^{\prime \prime}(B(1))\left(B^{\prime}(1)\right)^{2}+A^{\prime}(B(1)) B^{\prime \prime}(1)+\langle s\rangle \\
& =\left\langle k^{2}\right\rangle\langle w\rangle^{2}-\langle k\rangle\langle w\rangle^{2}+\langle k\rangle\left\langle w^{2}\right\rangle .
\end{aligned}
$$

Therefore, we obtain the condition for pathogen persistence among the population:

$$
-\frac{\ln (1-\beta)}{\mu}>\left(\frac{\left\langle k^{2}\right\rangle\langle w\rangle}{\langle k\rangle}+\frac{\left\langle w^{2}\right\rangle-\langle w\rangle^{2}}{\langle w\rangle}\right)^{-1} .
$$

This implies that the infection probability $\beta$ necessary for the pathogen to persist in the system is a decreasing function of the heterogeneity of either the weight or the degree distribution, or both.

\section{Numerical simulations}

The analytical results were further analyzed by performing numerical simulations of epidemic spreading on annealed networks generated by exploring $\left(\gamma_{k}, \gamma_{w}\right)$. For each $\left(\gamma_{k}, \gamma_{w}, \beta, \mu\right)$ we simulate 100 epidemic scenarios unfolding on independently generated graphs, randomly choosing ten infected nodes as the initial condition (we arbitrarily chose the number of ten infected nodes as an initial condition since we want to explore the infected stable state more than the invasion pattern and 
we want to avoid an exceptionally high number of scenarios with disease extinction) and observing the steady state at the endemic equilibrium from which we evaluate the fraction of infected nodes $\langle i\rangle$. Since the equilibrium of a SIS model in the active phase is not unequivocally defined, we stated that an epidemic spreading reaches the equilibrium at time $t$ if the median of the prevalences $i(\tau)$ with $\tau \in[t-50, t]$ was within the $1 \%$ percentile of the prevalences $i(\tau)$ with $\tau \in[t-100, t-50]$. In addition, it is also worth to stress that the equilibria (disease-free or not) were reached long before the maximum allowed time, $t_{\max }=10^{4}$.
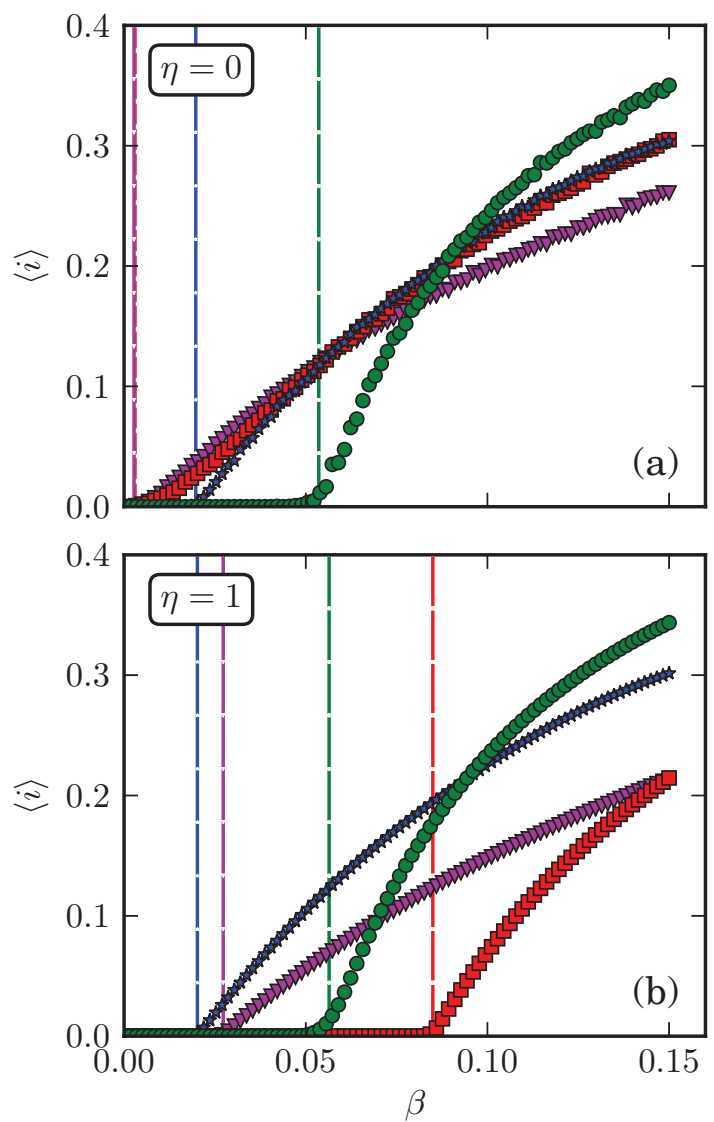

$$
\begin{array}{|ccc}
\nabla & \nabla & \gamma_{k}=0, \gamma_{w}=0 \\
\square & \square & \gamma_{k}=1, \gamma_{w}=0 \\
\star \quad & \star & \gamma_{k}=0, \gamma_{w}=1 \\
\bullet & \bullet & \gamma_{k}=1, \gamma_{w}=1
\end{array}
$$

FIG. 4. (Color online) Fraction of infected nodes at endemic equilibrium reached as function of $\beta$, the transmission probability. For this figure $\mu$ is fixed to 1 . (a) Results on annealed networks with stable weight pattern, $\eta=0$. (b) Results on annelead networks with dynamical assignment of weights, $\eta=1$. Recovery probability $\mu$ is fixed to 1 . Vertical lines show the theoretical expectation of the thresholds. On graphs associated with $\eta=0$ the heterogeneity of both edge weights and node degrees seems to support pathogen persistence. On the other hand, on networks with dynamical assignment of weights, $\eta=1$, infection stability is more often achieved on graphs with homogeneously distributed weights.
Numerical simulations reported at the top of Fig. 4 show the influence of a broad strength distribution (i.e., $\gamma_{k}=0$ or $\left.\gamma_{w}=0\right)$ for the pathogen persistence. In particular, this picture shows the strong impact of the weight heterogeneity on the critical transmission parameter. Figure 4 also shows the good match between theoretical and empirical epidemic thresholds on annealed graphs. We underline that magenta and red lines seem almost coincident but the red one is in fact slightly larger than the magenta, consistent with the observed epidemic curves. Empirical results on quenched networks show similar patterns in terms of threshold effects for the different scenarios (data not shown) but, as expected, the HMF approximation does not provide a good estimation of the $\beta_{c}$.

We integrate the aforementioned disease spreading with a hyperbolic variant of the Brent method for root finding [62]. By using a tolerance $10^{-2}$ we detect the $\beta_{c}$ for which $\langle i\rangle$ is equal to 0.05 . $\langle i\rangle$ is measured as the averaged fraction of infected nodes at infection persistence in 100 graphs. We explore $\mu=0.25$ and $\mu=1$ as the probability of recovery. Although the analytical description matches fairly well with the numerical simulations, it is worthwhile to emphasize that our interest lies in the qualitative investigation of $\beta_{c}$ for a wide range of networks with increasing heterogeneity rather than providing a quantitative estimate of the critical point. In Fig. 5 we show the surface $\beta_{c}\left(\gamma_{k}, \gamma_{w}\right)$ obtained for annealed networks. Results confirm the well-known behavior $[9,11,63]$ of epidemic spreading on graphs: the larger the heterogeneity of connections among nodes, the lower the transmission probability needed for pathogen persistence. Moreover, in agreement with the analytical insights, the heterogeneity of weights also fosters pathogen persistence. We refer to the top picture of Fig. 5 for an overview of these results.

\section{DYNAMIC ASSIGNMENT OF WEIGHTS}

\section{A. Graph generation}

As a second step, we investigated how the dynamic assignment of weights on edges (i.e., when edges change their weight at each time step) influences infection spreading. In particular, given a network (quenched or annealed) defined by a degree and weight sequence originated by couple $\left(\gamma_{k}, \gamma_{w}\right)$ as in Sec. II A, at each time step we permute a fraction $\eta \in[0,1]$ of weights. To provide the reader additional insights about the effect of this procedure, we plot in Fig. 6 the strength of a prototypical medium-highly connected node of degree 50 in $\left(\gamma_{k}=0, \gamma_{w}=\{0,0.8,1\}\right)$ networks with $\eta=1$. In a 300-step large time window sudden spikes in the node's strength can be observed and these are caused by the re-assignation of weights on edges.

We further explore the fluctuations of link properties as a consequence of the weight shuffling. We consider the evolution rates of edge weights,

$$
\ln \left(\frac{w_{i j}(t+1)}{w_{i j}(t)}\right)
$$

for different values of $\eta$. The distributions of those evolution rates are shown in Fig. 7 and it is worth noticing that they resemble many real-world systems $[53,64,65]$ where the evolution rate distribution shows an exponentially decaying 
tail. Not surprisingly, the probability of a high evolution rate increases based on the fraction of edge weights reallocated, $\eta$. It is interesting to note that even for a small fraction of permuted weights (e.g., $\eta=0.3$ ) the weight of an edge could dramatically change, thus generating a bursted behavior.

\section{B. Disease spreading}

\section{Analytical description for $\eta=1$}

Considering the complete dynamical case, i.e., $\eta=1$, we provide analytical insights about the epidemic threshold on annealed graphs. At each time step the weights of edges are given according to $q(w)$, i.e., the weight probability distribution, and with $\gamma_{w}$, i.e., the parameter responsible for tuning the heterogeneity of the distribution. Furthermore, assuming no correlation between the degree of nodes and the edge weights connecting them, and since the weights on edges are allocated at each time step, we could distinguish nodes according to their degree, i.e., using degree block approximation. Therefore, the probability that a susceptible node connected with an infective one will be infected is

$$
\mathbb{P}=1-\left[\sum_{w}(1-\beta)^{w} q(w)\right] .
$$
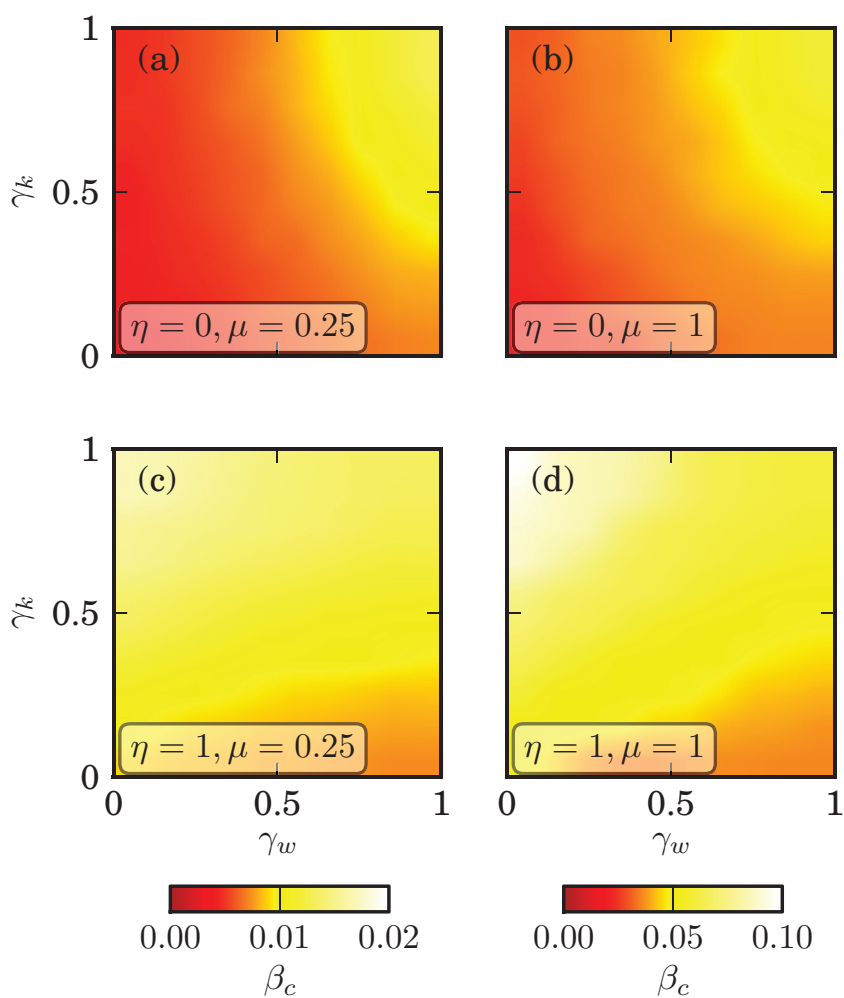

FIG. 5. (Color online) Estimated epidemic threshold $\beta_{c}$ as function of $\gamma_{k}$ and $\gamma_{w}$ on annealed networks. (a)-(c) Recovery probability is $\mu=0.25$; (b)-(d) we fix $\mu=1$. (a),(b) Results obtained for graphs with stable weights pattern, $\eta=0$; (c),(d) results obtained on graphs with dynamical assignment of weights, $\eta=1$. By comparing these two rows, a clear difference of behavior could be detected: for $\eta=1$ the edge weight heterogeneity ceases to be a favorable condition for infection spreading. We further explore this behavior in Fig. 10.

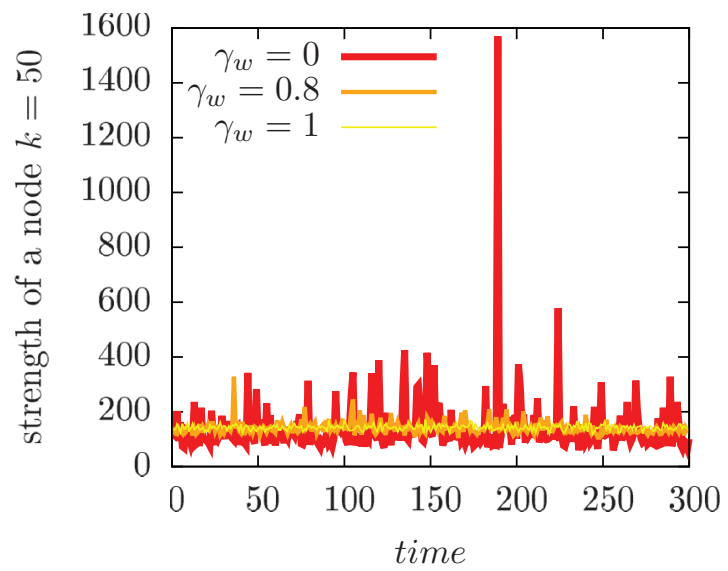

FIG. 6. (Color online) Strength of a randomly chosen node of degree 50 in $\left(\gamma_{k}=0, \gamma_{w}=\{0,0.8,1\}\right)$ networks with $\eta=1$ fraction edge weights permuted at each time step.

Hence, the probability that a node of degree $k$ becomes infected is

$$
1-(1-\mathbb{P})^{k \Theta_{k}}
$$

where $\Theta_{k}$ is the probability that an edge connects a node of degree $k$ and an infective node. Assuming no degree correlation we have

$$
\Theta_{k}=\frac{1}{\langle k\rangle} \sum_{k^{\prime}} k^{\prime} p\left(k^{\prime}\right) i_{k^{\prime}}=\Theta,
$$

where $i_{k^{\prime}}$ is the fraction of infective nodes of degree $k^{\prime}$. Now, translating Eq. (1) in degree block approximation and following a similar approach to what we have done before, we obtain the threshold condition necessary for disease invasion:

$$
-\frac{\ln (1-\mathbb{P})}{\mu}>\frac{\langle k\rangle}{\left\langle k^{2}\right\rangle} .
$$

Below, we further explore result of Eq. (2) by applying it to two fat-tailed probability distributions of edge weights.

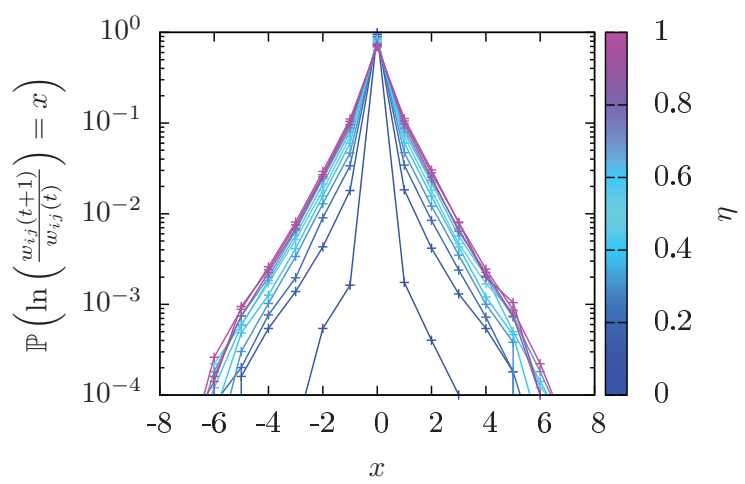

FIG. 7. (Color online) Probability density of the evolution rate of edge weights for dynamic heterogeneous networks $\left(\gamma_{k}=0, \gamma_{w}=0\right)$ for different $\eta$ values. $\eta$ ranges from 0 , blue (lower) curve, to 1 , magenta (upper) curve. 


\section{Instantaneous weight assignment, power-law distributed}

Assuming that the instantaneous weight of edges follows a power-law distribution, $q(w)=A w^{-\alpha}$, where $A$ is the normalizing constant, we could explicitly write $\mathbb{P}$ as

$$
\mathbb{P}=1-A \operatorname{Li}_{\alpha}(1-\beta),
$$

where $\mathrm{Li}_{n}(x)$ is the $n$th polylogarithm of $x$. Now, since

$$
\frac{\partial \operatorname{Li}_{n}(x)}{\partial n}=-\sum_{k} \frac{x^{k}}{k^{n}} \ln (k)<0
$$

and

$$
\frac{\partial \operatorname{Li}_{n}(x)}{\partial x}=\frac{\operatorname{Li}_{n-1}(x)}{x}>0,
$$

we have that both $\partial_{\alpha} \mathbb{P}$ and $\partial_{\beta} \mathbb{P}$ are larger than zero, or in other words we have that $\mathbb{P}$ is a growing function of both of its variables $\beta$ and $\alpha$. Applying such knowledge to Eq. (2) we conclude that as $\alpha$ increases, so does the homogeneity of instantaneous weights, while the transmission probability necessary for pathogen persistence decreases.

\section{Instantaneous weight assignment, negative-binomial distributed}

Assuming that the instantaneous weight of edges follows a negative-binomial distribution, $p(w)=\left(\begin{array}{c}w+r-1 \\ w\end{array}\right)(1-p)^{r} p^{w}$, with $p \in(0,1)$ and $r>0$, we could write $\mathbb{P}$ as

$$
\mathbb{P}=1-\left[\frac{1-p}{1-p(1-\beta)}\right]^{r} \text {. }
$$

Now, since we are interested in exploring the behavior of the epidemic for decreasing heterogeneity of instantaneous weight, we impose $r=\frac{\langle w\rangle(1-p)}{p}$. Therefore,

$$
\left\langle w^{2}\right\rangle=\langle w\rangle\left(\langle w\rangle+\frac{1}{1-p}\right)
$$

or in other words, for fixed $\langle w\rangle,\left\langle w^{2}\right\rangle$ increases as $p$ increases. Applying the change of variable just described in Eq. (3) the condition for pathogen persistence, Eq. (2), could be simplified as

$$
-\frac{1-p}{p} \ln \left(\frac{1-p}{1-p(1-\beta)}\right)>\mu \frac{\langle k\rangle}{\left\langle k^{2}\right\rangle\langle w\rangle} .
$$

Now, defining $H(p, \beta)$ as the left hand of the previous inequality, its partial derivatives are

$$
\partial_{\beta} H(p, \beta)=\frac{1-p}{1-p(1-\beta)}>0
$$

and

$$
\begin{aligned}
\partial_{p} H(p, \beta) & \\
= & \frac{1}{p^{2}}\left[\ln \left(\frac{1-p}{1-p(1-\beta)}\right)+\frac{\beta p}{1-p(1-\beta)}\right] \\
& <\frac{1}{p^{2}}\left[\left(\frac{1-p}{1-p(1-\beta)}\right)-1+\frac{\beta p}{1-p(1-\beta)}\right]<0 .
\end{aligned}
$$

Thus, we conclude that the smaller the value of $p$, the larger the homogeneity of instantaneous weights, and the smaller the transmission probability needed for the pathogen to spread.

\section{Numerical simulations}

It is already known, [25,26,36,66], that the temporal dynamic of a network reduces the probability of pathogen invasion. Our numerical simulations, shown in Fig. 4 for annealed graphs confirm this result: when all weights are reshuffled at each time step, $\eta=1$, the stable infected state is reached for larger transmission probability if compared to what happens on graphs with weights that remain constant with respect to time, $\eta=0$. It is also worthwhile to stress that the epidemic curves obtained for $\gamma_{w}=1$ do not change comparing $\eta=0$ with $\eta=1$. This is due to the fact that when the weights are homogeneously distributed only little changes could be observable with dynamical behavior.

Consistently with the analytical results, epidemic simulations show that in the dynamical case the homogeneity of edge weights is a favorable condition for infection spreading, conversely to what happens in the static case. Performing extensive numerical simulations in the whole $\left(\gamma_{k}, \gamma_{w}\right)$ domain, we explore how the parameter $\eta$, governing the dynamic
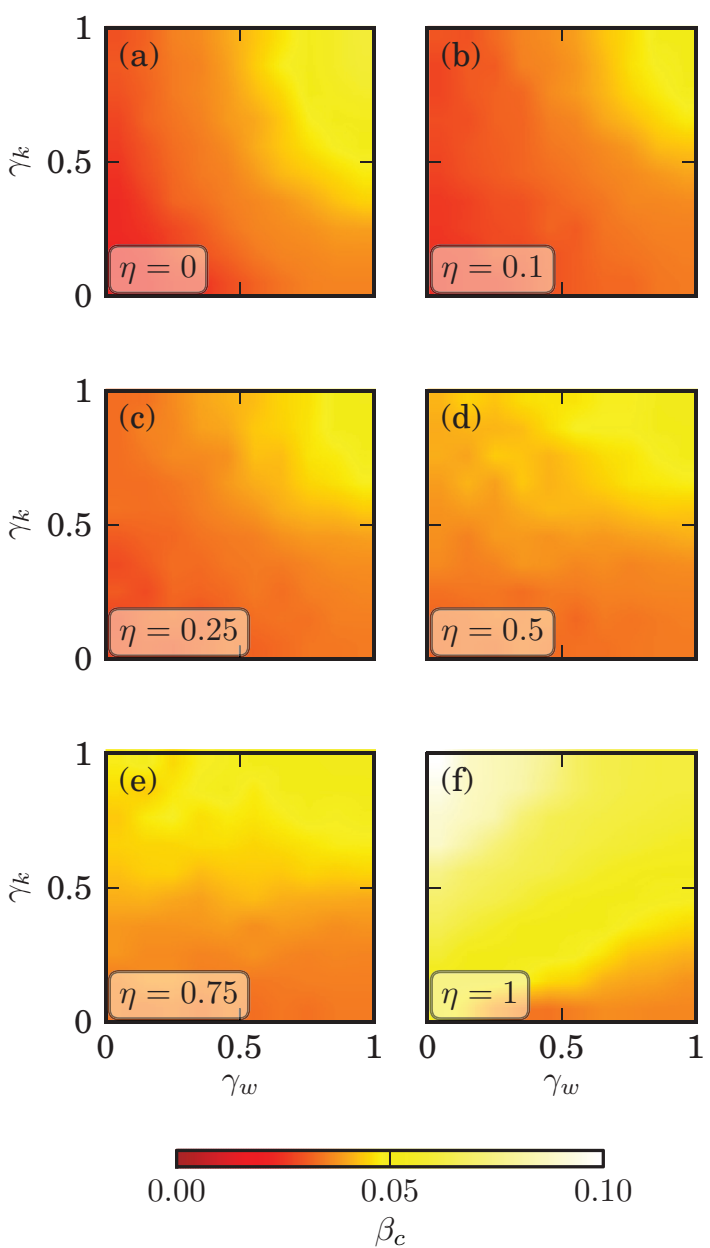

FIG. 8. (Color online) Critical values of transmission probability $\beta_{c}$ for different fractions of dynamically assigned weights $\eta$ in annealed graphs. Recovery probability $\mu$ is fixed to 1 . 

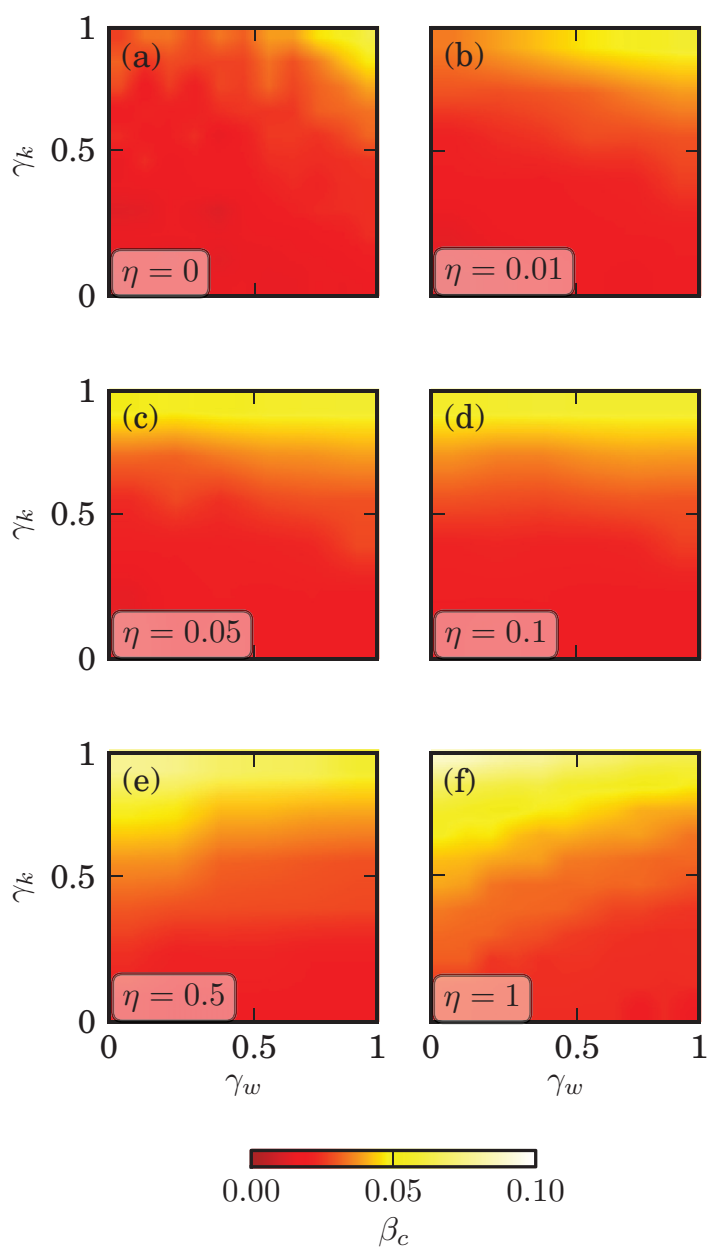

FIG. 9. (Color online) Critical values of transmission probability $\beta_{c}$ for different fractions of dynamically assigned weights $\eta$ in quenched graphs. Recovery probability $\mu$ is fixed to 1 .

reallocation of weights, influences the spreading dynamics. Similarly to the static case, we depict the surface $\beta_{c}\left(\gamma_{k}, \gamma_{w}\right)$ for $\eta=1$. As recovery probability we explore $\mu=0.25$ and $\mu=1$. The patterns related to a sustained disease transmission drastically change if compared with results obtained with stable weights $(\eta=0)$, as shown in Fig. 5 for annealed networks. Since in the two extreme cases $(\eta=0, \eta=1)$ the epidemic thresholds show opposite trends, we also explore $\eta$ by investigating some intermediate regimes for annealed graphs in Fig. 8 (and for quenched in Fig. 9). Then, for each surface we linearly fit the curve $\beta_{c}\left(\gamma_{k}=1, \gamma_{w}\right)$ by a least-squares method. We plot on Fig. 10 the slope of the linear fit as a function of $\eta$. Indeed, a positive slope indicates a scenario in which the edge weight heterogeneity positively interacts with the infection persistence while a negative slope means the edge weight heterogeneity hinders disease spreading. Results are plotted in Fig. 10 where we recover the qualitative results of the previously discussed scenarios with $\eta=0$ and $\eta=1$ and the transient behavior between the two regimes. Curves obtained for quenched and annealed networks are different due to the different response of the two systems to changing $\eta$. In fact, annealed graphs are less susceptible to topology modifications, having a greater level of randomness if compared to quenched

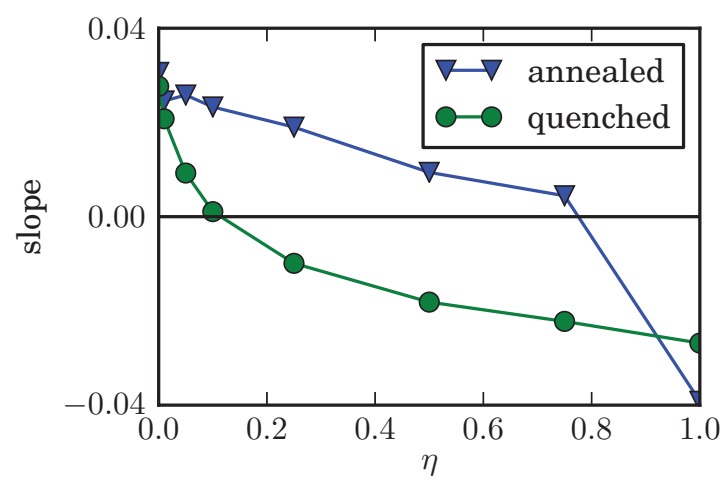

FIG. 10. (Color online) Slope of linear fitting of $\beta_{c}\left(\gamma_{k}=1, \gamma_{w}\right)$ for various $\eta$. Recovery probability $\mu$ is fixed to 1 .

networks. Sensitivity analysis performed on $\mu$ confirms that the pattern does not change if not for a scale factor. This scale factor should come as no surprise, since a lower recovery probability makes it easier for the pathogen persist in the population.

\section{CONCLUSIONS}

This work emphasizes the role of the weights and of their dynamic assignment to edges on the infection diffusion. As a general result we found that the evolving nature of weights on a graph is a limiting condition for the stability of infection. We suggest that this possibly happens when the graph evolution affects the role of hub nodes in the graph.

Our results show that for weighted networks the pathogen spreading capability is enhanced by heterogeneity of both degree and weight distributions. On the contrary, this tendency is reversed when the weights are dynamically assigned on edges at every time step. In particular, simulations show that the heterogeneity of weights acts to slow pathogen diffusion when we increase the fraction of edge weights that is dynamically assigned. Results suggest that more temporarily stable and heavier weights all over the network are better for pathogen spreading than some spikes of super-heavy links and a vast majority of very light weights where the transmission is very unlikely to occur. These results demonstrate the importance of dynamic behavior and edge weight distribution as features that should be taken into account when modeling infection transmissions. We strengthen our empirical results by analytical investigations. By using a HMF approach we were able to grasp the nature of the threshold $\beta_{c}$. Our result corroborates findings of previous work [52], indicating that the homogeneous assumption on the assignment of edge weights may produce an entirely different behavior of epidemic spreading than that observed with the heterogeneous assumption.

In the context of the cattle trade movements, our results could outline some fascinating suggestions. Let us consider the case of an infectious disease spreading among cattle by taking advantage of their trade movements (e.g., the foot-andmouth disease [67] or the diseases caused by bovine diarrhea virus [68]). It would be of interest to understand whether it would be a manageable and feasible containment policy for a state or superstate organization to intervene on commerce and impose a policy capable of driving the market to be 
more heterogeneous. This should decrease the probability of invasion and persistence, and it could be a preferable measure compared to a complete shutdown of the trade system. Extensive research in this direction will be the subject of future research.

Future work will be devoted to the analytical exploration of spreading on quenched networks. Working towards this goal, it might be useful to extend this framework to some real-world epidemic scenarios, also taking into account more complex transmission routes than SIS. An important extension to our research would be to integrate into our framework degree, weights, and temporal correlations as observed in real-world systems.

\section{ACKNOWLEDGMENTS}

Authors thank B. N. Iotti for fruitful discussions. L.F. acknowledges support from the Lagrange Project, CRT. and ISI Foundation. P.B. and M.G. acknowledge Compagnia di San Paolo and M.G. acknowledges local research funding of the University of Torino.
[1] S. N. Dorogovtsev, A. V. Goltsev, and J. F. F. Mendes, Rev. Mod. Phys. 80, 1275 (2008).

[2] C. Castellano and R. Pastor-Satorras, Phys. Rev. Lett. 96, 038701 (2006).

[3] C. Castellano and R. Pastor-Satorras, Phys. Rev. Lett. 105, 218701 (2010).

[4] Y. Wang, D. Chakrabarti, C. Wang, and C. Faloutsos, in Proceedings of the 22nd International Symposium on Reliable Distributed Systems, 2003 (IEEE, Piscataway, NJ, 2003).

[5] F. Chung, L. Lu, and V. Vu, Proc. Natl. Acad. Sci. USA 100, 6313 (2003).

[6] B. Ad. Prakash, D. Chakrabarti, M. Faloutsos, N. Valler, and C. Faloutsos, arXiv:1004.0060.

[7] S. Gómez, A. Arenas, J. Borge-Holthoefer, S. Meloni, and Y. Moreno, Europhys. Lett. 89, 38009 (2010).

[8] A. L. Lloyd and R. M. May, Science 292, 1316 (2001).

[9] R. Pastor-Satorras and A. Vespignani, Phys. Rev. Lett. 86, 3200 (2001).

[10] S. Bansal, B. T. Grenfell, and L. A. Meyers, J. R. Soc., Interface 4, 879 (2007).

[11] A. Barrat, M. Barthélemy, and A. Vespignani, Dynamical Processes on Complex Networks (Cambridge University Press, Cambridge, 2008).

[12] L. Freeman, Social Networks 1, 215 (1978).

[13] N. E. Friedkin, Am. J. Sociol. 96, 1478 (1991).

[14] G. Sabidussi, Psychometrika 31, 581 (1966).

[15] C. Dangalchev, Physica A: Stat. Mech. Appl. 365, 556 (2006).

[16] P. Bonacich, J. Math. Sociol. 2, 113 (1972).

[17] S. Brin and L. Page, Comput. Networks ISDN Syst. 30, 107 (1998).

[18] M. Kitsak, L. K. Gallos, S. Havlin, F. Liljeros, L. Muchnik, H. E. Stanley, and H. A. Makse, Nat. Phys. 6, 888 (2010).

[19] A. Garas, F. Schweitzer, and S. Havlin, New J. Phys. 14, 083030 (2012).

[20] C. Castellano and R. Pastor-Satorras, Sci. Rep. 2, 371 (2012).

[21] P. Holme and J. Saramäki, Phys. Rep. 519, 97 (2012).

[22] Temporal Networks, edited by P. Holme and J. Saramäki (Springer, Heidelberg, 2013).

[23] Dynamics on and of Complex Networks, edited by A. Mukherjee, F. Peruani, M. Choudhury, N. Ganguly, and B. Mitra (Birkhäuser, Boston, 2013), Vol. 2.

[24] R. Pfitzner, I. Scholtes, A. Garas, C. J. Tessone, and F. Schweitzer, Phys. Rev. Lett. 110, 198701 (2013).
[25] L. E. Rocha, A. Decuyper, and V. D. Blondel, in Dynamics On and Of Complex Networks, edited by A. Mukherjee, F. Peruani, M. Choudhury, N. Ganguly, and B. Mitra (Ref. [23]), Vol. 2, pp. 301-314.

[26] Y. Schwarzkopf, A. Rákos, and D. Mukamel, Phys. Rev. E 82, 036112 (2010)

[27] E. Volz and L. A. Meyers, Proc. R. Soc. London, Ser. B 274, 2925 (2007).

[28] E. Volz and L. A. Meyers, J. R. Soc. Interface 6, 233 (2009).

[29] J. C. Miller, A. C. Slim, and E. M. Volz, J. R. Soc. Interface 9, 890 (2012).

[30] T. Gross, C. J. D. D’Lima, and B. Blasius, Phys. Rev. Lett. 96, 208701 (2006).

[31] T. Gross and B. Blasius, J. R. Soc. Interface 5, 259 (2008).

[32] S. Van Segbroeck, F. C. Santos, and J. M. Pacheco, PLoS Comput. Biol. 6, e1000895 (2010).

[33] V. Marceau, P.-A. Noël, L. Hébert-Dufresne, A. Allard, and L. J. Dubé, Phys. Rev. E 82, 036116 (2010).

[34] M. Taylor, T. J. Taylor, and I. Z. Kiss, Phys. Rev. E 85, 016103 (2012).

[35] N. Masuda, K. Klemm, and V. M. Eguíluz, Phys. Rev. Lett. 111, 188701 (2013).

[36] N. Perra, B. Gonçalves, R. Pastor-Satorras, and A. Vespignani, Sci. Rep. 2, 469 (2012).

[37] Y. Gang, Z. Tao, W. Jie, F. Zhong-Qian, and W. Bing-Hong, Chin. Phys. Lett. 22, 510 (2005).

[38] S. Meloni, A. Arenas, and Y. Moreno, Proc. Natl. Acad. Sci. USA 106, 16897 (2009).

[39] A. Baronchelli and R. Pastor-Satorras, Phys. Rev. E 82, 011111 (2010).

[40] M. Deijfen, Math. Biosci. 232, 57 (2011).

[41] X. Chu, Z. Zhang, J. Guan, and S. Zhou, Physica A 390, 471 (2011).

[42] T. Britton, M. Deijfen, and F. Liljeros, J. Stat. Phys. 145, 1368 (2011).

[43] T. Britton and D. Lindenstrand, Math. Biosci. 240, 124 (2012).

[44] Z. Yang and T. Zhou, Phys. Rev. E 85, 056106 (2012).

[45] C. Kamp, M. Moslonka-Lefebvre, and S. Alizon, PLoS Comput. Biol. 9, e1003352 (2013).

[46] G. Miritello, E. Moro, and R. Lara, Phys. Rev. E 83, 045102 (2011).

[47] C. Cattuto, W. Van den Broeck, A. Barrat, V. Colizza, J.-F. Pinton, and A. Vespignani, PLoS One 5, e11596 (2010).

[48] P. Bajardi, A. Barrat, L. Savini, and V. Colizza, J. R. Soc. Interface 9, 2814 (2012). 
[49] L. E. C. Rocha, F. Liljeros, and P. Holme, PLoS Comput. Biol. 7, e1001109 (2011).

[50] L. E. C. Rocha and V. D. Blondel, PLoS Comput. Biol. 9, e1002974 (2013).

[51] K. Eames, J. Read, and W. Edmunds, Epidemics 1, 70 (2009).

[52] J. Stehle, N. Voirin, A. Barrat, C. Cattuto, V. Colizza, L. Isella, C. Regis, J.-F. Pinton, N. Khanafer, W. Van den Broeck, and P. Vanhems, BMC Med. 9, 87 (2011).

[53] P. Bajardi, A. Barrat, F. Natale, L. Savini, and V. Colizza, PLoS One 6, e19869 (2011).

[54] A. Machens, F. Gesualdo, C. Rizzo, A. Tozzi, A. Barrat, and C. Cattuto, BMC Infect. Dis. 13, 185 (2013).

[55] G. Miritello, R. Lara, M. Cebrian, and E. Moro, Sci. Rep. 3, 1950 (2013).

[56] A. Barrat, M. Barthlemy, R. Pastor-Satorras, and A. Vespignani, Proc. Natl. Acad. Sci. U.S.A. 101, 3747 (2004).

[57] J. Moreno, Who Shall Survive? Foundations of Sociometry, Group Psychotherapy, and Sociodram (Beacon House, New York, 1934).
[58] M. Molloy and B. Reed, Random Struct. Alg. 6, 161 (1995).

[59] M. Catanzaro, M. Boguñá, and R. Pastor-Satorras, Phys. Rev. E 71, 027103 (2005).

[60] R. Anderson and R. May, Infectious Diseases of Humans: Dynamics and Control (Oxford University Press, Oxford, 1992).

[61] H. S. Wilf, Generating Functionology, 3rd ed. (K. Peters, Wellesley, Massachusetts, 2006).

[62] R. P. Brent, Algorithms for Minimization without Derivatives (Prentice-Hall, Englewood Cliffs, NJ, 1973).

[63] R. Pastor-Satorras and A. Vespignani, Phys. Rev. E 63, 066117 (2001).

[64] M. H. R. Stanley, L. A. N. Amaral, S. V. Buldyrev, S. Havlin, H. Leschhorn, P. Maass, M. A. Salinger, and H. E. Stanley, Nature (London) 379, 804 (1996).

[65] A. Gautreau, A. Barrat, and M. Barthlemy, Pro. Natl. Acad. Sci. 106, 8847 (2009).

[66] M. Karsai, M. Kivelä, R. K. Pan, K. Kaski, J. Kertész, A.-L. Barabási, and J. Saramäki, Phys. Rev. E 83, 025102 (2011).

[67] M. J. Keeling, Proc. R. Soc. London, Ser. B 272, 1195 (2005).

[68] M. Tinsley, F. Lewis, and F. Brulisauer, Vet. Res. 43, 11 (2012). 